\title{
Toxic epidermal necrolysis - an investigation to dye for?
}

\begin{abstract}
We report the first case in Australia, as confirmed by the Therapeutic Goods Administration, of toxic epidermal necrolysis associated with the iodinated contrast medium iopamidol. It serves as a warning about the use of contrast in imaging and cardiac catheterisation and a reminder of the need for increased awareness of the issue.
\end{abstract}

A 44-year-old woman presented to the emergency department with a 3-day history of a progressive rash, fever, malaise and mucosal ulceration. She met the diagnosis of toxic epidermal necrolysis (TEN) based on the following criteria: bullae and desquamation affecting about $84 \%$ of the body surface (Box 1 and Box 2), buccal and vaginal ulceration, a positive Nikolsky sign (this is a useful sign in bullous skin diseases and can be demonstrated by rubbing the skin surface, which will blister within a few minutes if the sign is positive), fever, tachycardia and mild hypotension. She also had abnormal results of liver function tests: bilirubin level, $69 \mu \mathrm{mol} / \mathrm{L}$ (reference interval [RI], <20 $\mathrm{mol} / \mathrm{L}$ ); alkaline phosphatase level, 180U/L (RI, 25-100U/L); $\gamma$-glutamyl transferase level, 499 U/L (RI, <30 U/L); alanine aminotransferase level, $1730 \mathrm{U} / \mathrm{L}(\mathrm{RI},<30 \mathrm{U} / \mathrm{L})$; and aspartate aminotransferase level, 638U/L (RI, <30U/L). She had a white cell count of $4.3 \times 10^{9} / \mathrm{L}\left(\mathrm{RI}, 4.0-10.0 \times 10^{9} / \mathrm{L}\right)$ and a raised C-reactive protein level of $53.1 \mathrm{mg} / \mathrm{L}(\mathrm{RI},<5 \mathrm{mg} / \mathrm{L})$.

The patient was immediately transferred to the burns unit and managed with nanocrystalline silver dressings, intravenous immunoglobulin, aggressive fluid and electrolyte balance therapy, analgesia and intravenous antibiotics. She was discharged home on Day 15.

Histopathological examination (Box 3) showed extensive epidermal necrosis and subepidermal clefting with a sparse superficial perivascular infiltrate of lymphocytes, occasional neutrophils and eosinophils, and exocytosis of cells into the epidermis. Results of staining for immunofluorescence were negative. This was consistent with the clinical diagnosis of TEN, and the possibility of pemphigus vulgaris was excluded.

Theresa Ly $\mathrm{BSc}(\mathrm{Adv}), \mathrm{MBBS}(\mathrm{Hons})$,
$\mathrm{MIPH}^{1}$

$\mathrm{MIPH}$

Nicholas Stewart $M B B S, B S C^{2}$

Stephen Lee $A M, D D M, F A C D^{3}$

1Concord Repatriation General Hospital, Sydney, NSW.

2 Skin and Cancer Foundation Australia Sydney, NSW.

3 University of Sydney, Sydney, NSW.

theresamly@gmail.com

doi: 10.5694/mjal4.00367

\section{Bullae and desquamation of back}

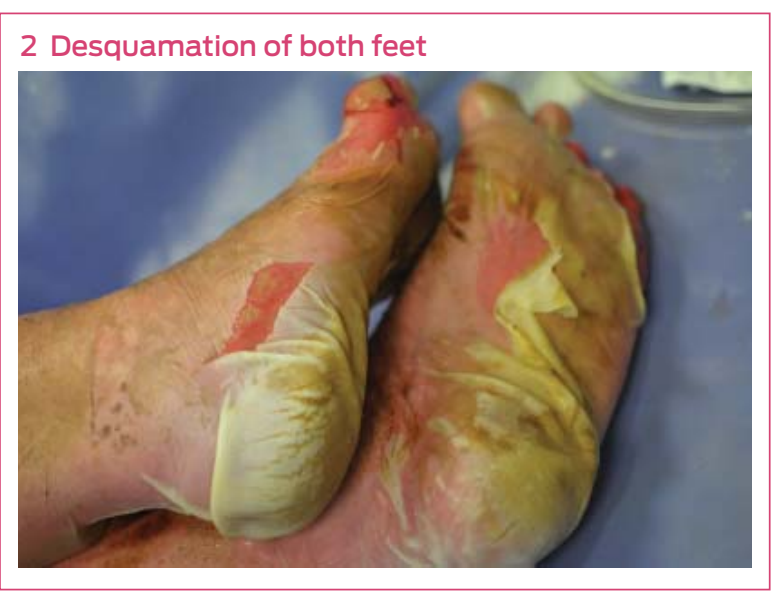

The patient's only recent exposure to medications included 150-200 $\mathrm{\mu g}$ of thyroxine sodium daily for 19 years and $2.5 \mathrm{mg}$ of indapamide daily for 6 months. The patient underwent a computed tomography (CT) neck scan with the contrast medium iopamidol about 4 weeks before the development of symptoms. The patient had recently (3 weeks before onset of rash) stopped taking a herbal "liver cleanser". She had been taking this intermittently for 2 months. She had no recent travel history or vaccinations.

The patient recalled having a previous CT scan of the neck before her surgery 17 years ago. Unfortunately, however, any records of this had been destroyed.

The patient's past medical history was notable for mild stable hypertension, hypothyroidism and a benign mixed salivary gland tumour electively excised 17 years earlier.

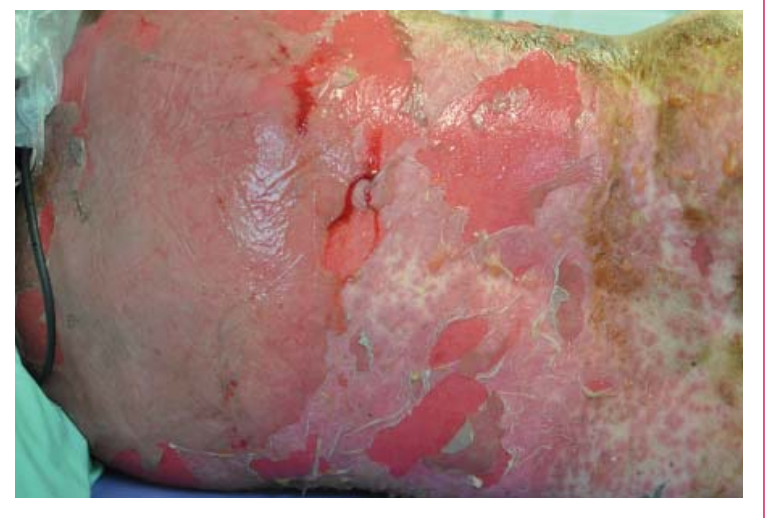

\section{Discussion}

Toxic epidermal necrolysis (TEN), or Lyell syndrome, is a rare and life-threatening severe systemic condition associated with dramatic cutaneous sloughing of up to $100 \%$ of the body surface area. The incidence of TEN is two cases per million person-years. ${ }^{1}$ It is characterised by necrosis and subsequent detachment of the epidermis from the dermis in more than $30 \%$ the body surface. If not treated and managed promptly, the consequences can be fatal; patients are vulnerable to infections and sepsis leading to death. The mortality associated with TEN is high, at $30 \%-40 \% .^{2}$

At the other end of the spectrum, and more common, are mild-to-moderate skin reactions to contrast media (CM). 
These include, in increasing severity, lichenoid reaction, erythema multiforme and Stevens-Johnson syndrome. ${ }^{3}$ Patients at risk of late skin reactions are those with a previous history of CM reactions.,

TEN is attributed to medications in $80 \%$ of cases. ${ }^{1}$ The most commonly associated medications include sulfonamides, penicillin and other antibiotics, anticonvulsants, oxicam nonsteroidal anti-inflammatory drugs, allopurinol and corticosteroids. ${ }^{5}$ TEN commonly occurs 1-3 weeks after the start of therapy. Other triggers include infections, malignancy and vaccination. ${ }^{1}$

The dermatological reactions caused by CM can be classified as early or late reactions. Early reactions occur soon after injection of the contrast medium, and late reactions occur within a week. The incidence of late adverse reactions is $2 \%$. 3,6 They commonly present as maculopapular erythema, angioedema and urticaria. Evidence to date suggests that late reactions are more common with nonionic CM, in particular dimers, ${ }^{3}$ despite non-ionic CM being touted as having fewer adverse reactions. Late reaction incidence with non-ionic $\mathrm{CM}$ varies between $8 \%$ and $71 \%{ }^{3}$

Iodinated CM can be divided into ionic and non-ionic. The ionicity pertains to the osmolality the CM create in blood; ionic CM create higher osmolality leading to CM reaction. The move from the use of ionic $\mathrm{CM}$ to non-ionic $\mathrm{CM}$ was based on the need for an agent with fewer adverse effects and equal or slightly improved diagnostic efficacy. ${ }^{7}$ The morbidity and mortality associated with non-ionic $\mathrm{CM}$ were less than for ionic CM. ${ }^{8}$ Although non-ionic CM have these advantages, ionic CM are still in use today. A recent study highlighted that although non-ionic $\mathrm{CM}$ are the best tolerated in the early phase, they are associated with a higher level of adverse effects such as late skin reactions. ${ }^{8}$ Iopamidol is a non-ionic contrast medium.

There have been several cases of TEN caused by CM reported in the literature. Commonly, the cases have involved repeated exposure or sensitisation to the CM in the cardiac catheter laboratory over a period of days to even years. ${ }^{9-11}$ TEN occurs with subsequent exposures to the CM administered. In our case report, there was a history of prior CT scan of the neck; however, records of the scan are no longer held by the radiologist to verify the date and contrast medium used.

TEN has also been shown to be caused by gastrointestinal oral CM. ${ }^{12}$

Only two published cases of TEN have been attributed to the administration of iopamidol. The first case is of a young boy with subsequent exposure to iopamidol. ${ }^{10}$
3 Skin punch biopsy sample showing extensive epidermal necrosis (bracket) and subepidermal clefting (asterisk) with mixed inflammatory infiltrate and negative results of staining for immunofluorescence

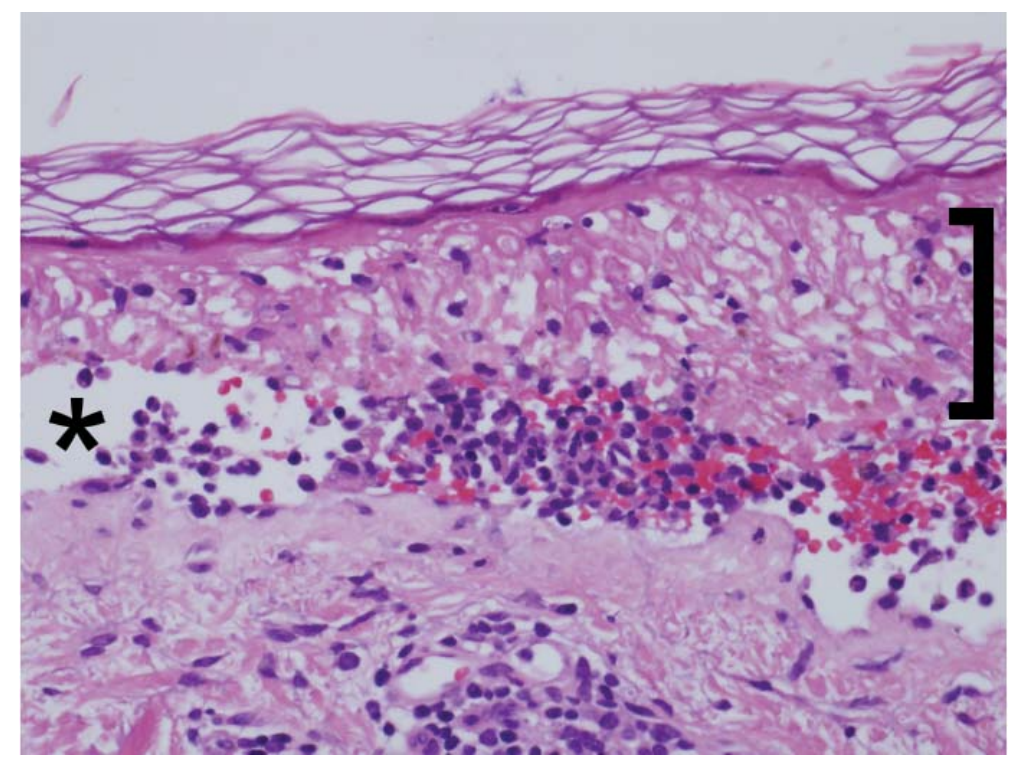

The case was not biopsy-proven TEN, but was based on clinical diagnosis. The second case is that of a patient who underwent intravenous urography for investigation of systemic lupus erythematosus with renal involvement. ${ }^{13}$ The patient died despite intensive care and support. Our case report would be the third reported case of TEN caused by iopamidol.

Although it is difficult to be sure that iopamidol was responsible for the development of TEN in our patient, it is highly likely to be the cause. She had been on indapamide for a period and had been taking the herbal "liver cleanser" intermittently. One recent case of herbal medicines and TEN has been reported..$^{14}$ However, as noted by the author, it was difficult to determine the causative agent.

It is important to be aware of the risk of CM and to think twice about the necessity of CM in imaging. Although rare, life-threatening adverse effects such as TEN should lead to reconsideration of contrast dyes, as patients may suffer unnecessarily or lose their lives.

Acknowledgements: We acknowledge the histopathological assistance provided by Charles Chan in this case report.

Competing interests: No relevant disclosures. 
1 Tartarone A, Lerose R. Stevens-Johnson syndrome and toxic epidermal necrolysis: what do we know? Ther Drug Monit 2010; 32: 669-672.

2 Borchers AT, Lee JL, Naguwa SM, et al. Stevens-Johnson syndrome and toxic epidermal necrolysis. Autoimmun Rev 2008; 7: 598-605.

3 Webb JA, Stacul F, Thomsen HS, Morcos SK; Members of the Contrast Media Safety Committee of the European Society of Urogenital Radiology. Late adverse reactions to intravascular iodinated contrast media. Eur Radiol 2003; 13: 181-184.

4 Thomsen H, Webb JAW. Contrast media. Safety issues and ESUR guidelines. 3rd ed. Heidelberg: Springer, 2013.

5 Ofoma UR, Chapnick EK. Fluconazole induced toxic epidermal necrolysis: a case report. Cases J 2009; 2: 9071.

6 Sedano E, Vega JM, Rebello S, et al. Delayed exanthema to nonionic contrast medium. Allergy 2001; 56: 1015-1016.

7 McClennan B. Ionic and nonionic iodinated contrast media: evolution and strategies for use. AJR Am J Roentgenol 1990; 155: 225-233.

8 Sutton AG, Finn P, Grech ED, et al. Early and late reactions after the use of iopamidol 340, ioxaglate 320, and iodixanol 320 in cardiac catheterization. Am Heart J 2001; 141: 677-683.
9 Baldwin BT, Lien MH, Khan H, Siddique M. Case of fatal toxic epidermal necrolysis due to cardiac catheterization dye. J Drugs Dermatol 2010; 9: 837-840.

10 Lee ML, Chiu IS. Toxic epidermal necrolysis incriminating iopamidol in a child after cardiac catheterization. Int J Cardiol 2002; 82: 95-97.

11 Rosado A, Canto G, Veleiro B, Rodríguez J. Toxic epidermal necrolysis after repeated injections of iohexol. AJR Am J Roentgenol 2001; 176: 262-263.

12 Schmidt BJ, Foley WD, Bohorfoush AR. Toxic epidermal necrolysis related to oral administration of diluted diatrizoate meglumine and diatrizoate sodium. AJR Am J Roentgenol 1998; 171: 1215-1216.

13 Savill JS, Barrie R, Ghosh S, et al. Fatal Stevens-Johnson syndrome following urography with iopamidol in systemic lupus erythematosus. Postgrad Med J 1988; 64: 392-394.

14 Hemmige V, Jenkins E, Lee JU, Arora VM. Toxic epidermal necrolysis (TEN) associated with herbal medication use in a patient with systemic lupus erythematosus. J Hosp Med 2010; 5: 491-493. 